\title{
Short Note \\ Inertial Forces from Earthquakes on a Hyperloop Pod
}

\author{
by Thomas H. Heaton
}

\begin{abstract}
High-speed transit (1300 km/hr) using pods traveling in evacuated tubes has been proposed. This Short Note addresses how earthquake ground shaking is changed when it is experienced by a high-speed pod that is confined to a track. In particular, earthquake motions can cause lateral deformations of the tube that cause centripetal forces in the pod. I discuss the nature of these forces for the cases of (1) a tube that crosses a fault offset, (2) a tube that is deformed by traveling waves in the Earth, and (3) a tube that resonates between fixed points (e.g., a simple bridge). I suggest several schemes to control the peak centripetal accelerations of the pod.
\end{abstract}

\section{Introduction}

Elon Musk has suggested that very high-speed transportation $(350 \mathrm{~m} / \mathrm{s}$ or $1300 \mathrm{~km} / \mathrm{hr})$ could be achievable using sealed pods that move within evacuated tubes (3.5 m diameter; Garber, 2012). Several companies are currently developing designs for such a system and it has been suggested that a hyperloop system may be appropriate for California. What are the dynamics of a high-speed vehicle moving in a fixed tube that is subject to earthquake motion? Obviously, bends in the tube will cause centripetal accelerations that are perpendicular to the tube. A pod traveling at velocity $V_{P}$ in a tube of radius of curvature $R$ experiences a centripetal acceleration $a_{\text {centripetal }}$ of

$$
a_{\text {centripetal }}=\frac{V_{P}^{2}}{R}
$$

If $V_{P}=350 \mathrm{~m} / \mathrm{s}$, then the radius of curvature that corresponds to $1 \mathrm{~g}$ is about $12 \mathrm{~km}$. What are the inertial accelerations of a high-speed pod traveling in a fixed tube that is elastically deforming in an earthquake? It seems that there are several different possibilities: (1) the tube is deformed in the immediate vicinity of a static fault offset, (2) the tube is deformed by the transient motion of traveling seismic waves, and (3) the tube is deformed by lateral resonances of the tube (e.g., the modes of a bridge). I discuss each of these issues separately.

\section{Accelerations Due to a Fault Offset}

Assume that there is a fault offset $D$ and that the static motion of the ground in the direction perpendicular to the tube is described by

$$
u_{y}^{g}=\frac{D}{\pi} \arctan \frac{x}{L}
$$

in which $y$ is perpendicular to the tube; $y$ can be either horizontal or vertical. $x$ is the distance along the tube such that $x=0$ at the fault trace, and $L$ is a scaling factor that determines the width of the deformation zone. The total width of the zone is on the order of $10 L$. If time $t$ is measured with respect to the time at which the pod intersects the fault, then the position of the pod is

$$
u_{y}^{P}(t)=\frac{D}{\pi} \arctan \frac{V_{P} t}{L}=\frac{D}{\pi} \arctan \tau,
$$

in which $\tau \equiv t / T_{L}$ and $T_{L} \equiv L / V_{P}$, which is the time it takes for the pod to traverse the deformation zone. If the position of the pod is $x_{P}=V_{P} t,-\infty<t<\infty$, then the pod's velocity perpendicular to the track is given by

$$
\begin{aligned}
\dot{u}_{y}^{P} & =\frac{D}{\pi}\left[1+(\tau)^{2}\right]^{-1} \frac{d \tau}{d t}=\frac{D}{\pi}\left[1+\left(\frac{t V_{P}}{L}\right)^{2}\right]^{-1} \frac{V_{P}}{L} \\
& =\left(\frac{D}{T_{L} \pi}\right)\left[1+\left(\frac{t}{T_{L}}\right)^{2}\right]^{-1} .
\end{aligned}
$$

Then, the acceleration is

$$
\ddot{u}_{y}^{P}=\left(\frac{-2 t D}{T_{L}^{3} \pi}\right)\left[1+\left(\frac{t}{T_{L}}\right)^{2}\right]^{-2}=\left(\frac{-2 D}{T_{L}^{2} \pi}\right) \tau\left[1+\tau^{2}\right]^{-2} .
$$

The acceleration directly above the fault crossing, $t=0$, is zero, and the maximum accelerations occur when

$$
\frac{d^{3} u_{y}^{P}}{d \tau^{3}}=0=\left(\frac{-2 D}{T_{L}^{2} \pi}\right)\left\{\frac{1}{\left(1+\tau^{2}\right)^{2}}-\frac{2 \tau^{2}}{\left(1+\tau^{2}\right)^{3}}\right\},
$$

or when

$$
1+\tau^{2}-2 \tau^{2}=0
$$

Therefore, the maximum accelerations occur when $\tau= \pm 1$, or

$$
\left.\ddot{u}_{y}^{P}\right|_{\max }= \pm \frac{D}{2 \pi T_{L}^{2}}= \pm \frac{D V_{P}^{2}}{2 \pi L^{2}} .
$$


The deformation length required to limit the acceleration to less than $\left.\ddot{u}_{y}^{P}\right|_{\max }$ is then

$$
L=V_{P} \sqrt{\frac{D}{\left.2 \pi \ddot{u}_{y}^{P}\right|_{\max }}} .
$$

If the speed of the pod is $350 \mathrm{~m} / \mathrm{s}$, then a maximum acceleration of $1 g$ occurs when $L \approx 44 \sqrt{D}$. For example, if the fault offset is $9 \mathrm{~m}$, then the maximum accelerations can be kept below $1 g$ by smoothly deforming the tube over a 1320-m-wide stretch spanning the fault. This may be feasible by employing a flexurally stiff tube that is mounted to stiff pylons with frictional bearings that can slide laterally. In this case, the mounting system would need to be able to slide up to $4.5 \mathrm{~m}$ in the vicinity of the fault crossing. Construction of a system to distribute fault offset over a length of pipeline was employed by the Alyeska Pipeline Service Company to distribute strain in the TransAlaska pipeline at the point where it intersects the Denali fault. The support system worked as intended during the 2002 M 7.9 Denali earthquake and, despite a $6 \mathrm{~m}$ offset of the pipeline, the pipeline deformation was sufficiently distributed to avoid rupture of the pipe (Honegger et al., 2004). In the case of the TransAlaska pipeline, the designers included an $S$ curve in the pipe that helped distribute strain during the offset. High centripetal forces would preclude the use of $S$ curves in a hyperloop system.

Although distributing the pipe deformation over the largest possible distances is dynamically desirable, cost issues may favor designs that minimize the width of the deformation zone. This suggests another strategy to deform the pipe at a fault crossing. That is, to force the pipe to bend in constant curvature sections that induce constant pod accelerations $\pm a_{0}$ that are in opposite directions on either side of the fault crossing. In this case, the acceleration of the pod can be described as

$$
\ddot{u}_{y}^{P}=a_{0}\left[\prod\left(\frac{t+\frac{1}{2}}{T_{0}}\right)-\prod\left(\frac{t-\frac{1}{2}}{T_{0}}\right)\right],
$$

in which $T_{0}$ is the time it takes the pod to traverse each of the curved sections and

$$
\prod(t) \equiv\left\{\begin{array}{ll}
1 & |t|<\frac{1}{2} \\
0 & |t|>\frac{1}{2}
\end{array} .\right.
$$

I can doubly integrate equation (10) to obtain

$$
u_{y}^{P}=a_{0}\left\{\begin{array}{ll}
0 & \frac{t}{T_{0}}<-1 \\
\frac{1}{2} t^{2} & -1<\frac{t}{T_{0}}<0 \\
\frac{1}{2}\left(t^{2}+T_{0}^{2}\right) & 0<\frac{t}{T_{0}}<1 \\
T_{0}^{2} & \frac{t}{T_{0}}>1
\end{array} .\right.
$$

That is, the total offset of the pipe across the deformation zone is $a_{0} T_{0}^{2}$. The total width of the deformation zone is $W=2 V_{P} T_{0}$, so the total offset can be written as $D=a_{0}\left(W / 2 V_{P}\right)^{2}$. Finally, the required width of the deformation zone is then obtained as a function of the fault offset and the pod acceleration, or $W=2 V_{P} \sqrt{D / a_{0}}$. If the fault slip is $9 \mathrm{~m}$ and the acceleration is $1 g$, then a $350 \mathrm{~m} / \mathrm{s}$ pod would require a total deformation width of $738 \mathrm{~m}$, which is substantially smaller than the $1320 \mathrm{~m}$ required by a pipe with an arctan deformation shape.

The analyses above can be used for any component of deformation perpendicular to the track. However, deforming the track into a smooth shape seems easier to accomplish if the motion is horizontal. That is, if there is significant vertical offset of the track at the Earth's surface, it may be difficult to design a system that minimizes tube curvature; if $L$ is small, the accelerations become extreme because they increase as $1 / L^{2}$.

\section{Tube Deformed by a Traveling Seismic Wave}

Assume that a pod is traveling in the $x$ direction at velocity $V_{P}$ and that the surface of the Earth is deformed by a wave for which the $y$ component of motion is given by a nondispersive wave that is also traveling in the $x$ direction with apparent velocity $c$. Then, the $y$ component of the ground displacement is $u_{y}^{g}(t-(x / c))$ (the $x$ component is irrelevant because the pod is not constrained in the $x$ direction). Now the $x$ position of the pod is just $x_{P}=t V_{P}$, so the $y$ component of the pod motion $u_{y}^{P}(t)$ is just

$$
u_{y}^{P}(t)=u_{y}^{g}\left(t-\frac{x_{P}}{c}\right)=u_{y}^{g}\left[t\left(1-\frac{V_{P}}{c}\right)\right] .
$$

Now, I can determine the $y$ component of the pod's velocity as

$$
\dot{u}_{y}^{P}(t)=\frac{d}{d t} u_{y}^{g}\left[t\left(1-\frac{V_{P}}{c}\right)\right]=\left(1-\frac{V_{P}}{c}\right) \dot{u}_{y}^{g}\left[t\left(1-\frac{V_{P}}{c}\right)\right] .
$$

Differentiating a second time, I obtain the acceleration

$$
\ddot{u}_{y}^{P}(t)=\left(1-\frac{V_{P}}{c}\right)^{2} \ddot{u}_{y}^{g}\left[t\left(1-\frac{V_{P}}{c}\right)\right] .
$$

Notice that

$$
\left.\ddot{u}_{y}^{P}(t)\right|_{V_{P} \rightarrow c}=0 .
$$

That is, if the pod is traveling at the apparent wavespeed, it experiences no acceleration. Of course, this solution is not physical because a pod that is traveling at the wavespeed would not experience the wave, because the wave would never catch up to the pod. If, on the other hand, the pod is traveling at the wavespeed but in the opposite direction from the wave propagation, then the acceleration on the pod would be four times larger than the ground acceleration. Notice that the timing of the acceleration experienced by the pod is compressed by a factor of $\left(1-\left(V_{P} / C\right)\right)$.

Although the pod can experience large accelerations if it travels into the propagating wavefield, the amplitudes of the 
transient displacements are unchanged by the pod's motion; it is just that these motions happen faster in the pod's reference frame.

Estimating the apparent velocity of the wave along the surface of the Earth is a common problem in seismology and it depends on the seismic velocities of the crust and the geometry of the earthquake relative to the station. An apparent horizontal wavespeed of $5000 \mathrm{~m} / \mathrm{s}$ would be typical for a shear wave located in the near-source region of damaging earthquakes. In this case, the velocity of a hyperloop pod is not large enough to cause significant amplification of the accelerations (factor of $0.5 \%$ ). However, short-period surface waves in basins can have group velocities of less than $300 \mathrm{~m} / \mathrm{s}$, and in this case the accelerations would be strongly affected (the particulars depend on a number of factors that can be calculated from standard seismological analysis).

\section{Accelerations from Standing Waves}

I can also calculate the accelerations of a pod traveling through a tube that is experiencing a standing harmonic wave (e.g., lateral modal resonance of a bridge, similar to the fundamental modal vibration of a tensioned string). Assume that the inertial motion on the bridge is the sum of the motion of the ground (the bridge pier) plus the deformation of the bridge. I assume that the bridge consists of the hyperloop pipe that is supported at both ends of the bridge. If the lateral motion of the pipe is harmonic, then I assume that

$$
u_{y}^{P}(x, t)=u_{y}^{g}(x, t)+A \cos \left(\omega_{0} t\right) \cos \left(k_{0} x\right),
$$

in which $A$ is the amplitude of the standing wave, and $\omega_{0}$ and $k_{0}$ are the natural frequency and wavenumbers of the fundamental-mode resonance, respectively. Assuming that the position of the pod is a linear function of time and that the inertial motion of the piers is approximately equal to the ground motion at the middle of the bridge, then equation (17) becomes

$$
u_{y}^{P}(t)=u_{y}^{g}(x=0, t)+A \cos \left(\omega_{0} t\right) \cos \left(k_{0} V_{P} t\right) .
$$

The corresponding velocity and acceleration is obtained by differentiating equation (18) with respect to time:

$$
\begin{aligned}
\dot{u}_{y}^{P}(x=0, t)= & \dot{u}_{y}^{g}(x=0, t)+A\left[-\omega_{0} \sin \left(\omega_{0} t\right) \cos \left(k_{0} V_{P} t\right)\right. \\
& \left.-\cos \left(\omega_{0} t\right) k_{0} V_{P} \sin \left(k_{0} V_{P} t\right)\right]
\end{aligned}
$$

$$
\begin{aligned}
\ddot{u}_{y}^{P}(x=0, t)= & \ddot{u}_{y}^{g}(x=0, t)+A\left[-\omega_{0}^{2} \cos \left(\omega_{0} t\right) \cos \left(k_{0} V_{P} t\right)\right. \\
& \left.+\omega_{0} \sin \left(\omega_{0} t\right) k_{0} V_{P} \sin \left(k_{0} V_{P} t\right)\right] \\
& +A\left[\omega_{0} \sin \left(\omega_{0} t\right)\left(k_{0} V_{P} t\right) \sin \left(k_{0} V_{P} t\right)\right. \\
& \left.-\cos \left(\omega_{0} t\right)\left(k_{0} V_{P}\right)^{2} \cos \left(k_{0} V_{P} t\right)\right] \\
= & \ddot{u}_{y}^{g}(x=0, t) \\
& -A\left(\omega_{0}^{2}+k_{0}^{2} V_{P}^{2}\right)\left[\cos \left(\omega_{0} t\right) \cos \left(k_{0} V_{P} t\right)\right] \\
& +2 \omega_{0} k_{0} V_{P}\left[\sin \left(\omega_{0} t\right) \sin \left(k_{0} V_{P} t\right)\right]
\end{aligned}
$$

The peak acceleration of the pod at the midpoint of the span occurs when $x=0$ and $t=0$, in which case (20) simplifies to

$$
\begin{aligned}
\ddot{u}_{y}^{P}(x=0, t=0)= & \ddot{u}_{y}^{g}(x=0, t)-A\left(\omega_{0}^{2}+k_{0}^{2} V_{P}^{2}\right) \\
& \approx \operatorname{SA}\left(\omega_{0}\right)\left(1+\frac{V_{P}^{2}}{c^{2}}\right),
\end{aligned}
$$

in which $c=\omega_{0} / k_{0}$ is the wavespeed for a flexural wave in a pipe, and $\operatorname{SA}\left(\omega_{0}\right) \equiv \max \left[\left(d^{2} / d t^{2}\right)\right.$ (position of bridge center in an inertial frame)] is the response spectral acceleration at $\omega_{0}$. The acceleration response of an oscillator vibrating at $\omega_{0}$ with a displacement amplitude of $A$ is approximately $\omega^{2} A$. The ratio of the acceleration of the moving pod to the stationary pod is then approximated as

$$
\frac{\mathrm{SA}_{\text {pod }}}{\mathrm{SA}_{\text {tube }}}=\left(1+\frac{k_{0}^{2} V_{P}^{2}}{\omega_{0}^{2}}\right)=\left(1+\frac{V_{P}^{2}}{c^{2}}\right) .
$$

In the case of a resonant beam of length $L$ that is fixed at its ends, the flexural wavespeed is related to the fundamental resonant period $T_{0}$ by $c=2 L / T_{0}$, so $\mathrm{SA}_{\text {pod }} / \mathrm{SA}_{\text {tube }}=$ $\left(1+\left(V_{P}^{2} T_{0}^{2} / 4 L^{2}\right)\right)$. Bernoulli-Euler beam theory can be used to determine the wavespeed of a harmonic bending wave $u_{y}=\cos (k x-\omega t)$, that is traveling along a cylindrical tube of inner and outer diameters of $d_{i}$ and $d_{o}$, respectively,

$$
c=\frac{2 \pi}{\lambda} \sqrt{\frac{E I}{\rho S}}
$$

(Housner and Vreeland, 1965, p. 331), in which $\lambda=2 L=$ $2 \pi / k_{0}$ is the wavelength of the standing wave, $E$ is Young's modulus, $\rho$ is material density, $S=\pi / 4\left(d_{o}^{2}-d_{i}^{2}\right)$, and $I=\pi / 64\left(d_{o}^{4}-d_{i}^{4}\right)$. Equation (23) can be simplified to

$$
\begin{aligned}
c & =\frac{\pi}{L} c_{L} \sqrt{\frac{I}{S}}=\frac{\pi}{L} c_{L} \sqrt{\frac{d_{0}^{4}-d_{i}^{4}}{16\left(d_{o}^{2}-d_{i}^{2}\right)}} \\
& =\frac{\pi}{L} c_{L} \sqrt{\frac{1}{16}\left(d_{o}^{2}+d_{i}^{2}\right)} \approx \frac{\pi d_{o}}{2 \sqrt{2} L} c_{L},
\end{aligned}
$$

in which I assume that the tube wall thickness is small compared with its diameter. I also identified that $c_{L}=\sqrt{E / \rho}$, which is the wavespeed of a longitudinal wave in a slender bar (about $5000 \mathrm{~m} / \mathrm{s}$ for steel). I can now estimate the period of the fundamental mode as

$$
T_{0}=2 \frac{L}{c}=\frac{4 \sqrt{2}}{\pi d_{o} c_{L}} L^{2} \approx \frac{1.8}{d_{o} c_{L}} L^{2} .
$$

Therefore, I can estimate the period of the $3.5 \mathrm{~m}$ steel tube to be $T_{0} \approx 1.03 \times 10^{-4} L^{2}$. That is, a $100 \mathrm{~m}$ span would have a period of about $1 \mathrm{~s}$, whereas a $200 \mathrm{~m}$ span would have a period of about $4 \mathrm{~s}$. The flexural wavespeed can now be written as a function of the span length, or

$$
c=2 \frac{L}{T_{0}}=\frac{\pi d_{o} c_{L}}{2 \sqrt{2} L} .
$$


I am now able to rewrite the ratio of the high-speed pod spectral acceleration relative to the stationary pod using equation (22),

$$
\begin{aligned}
\frac{\mathrm{SA}_{\text {pod }}}{\mathrm{SA}_{\text {tube }}}= & \left(1+\frac{V_{P}^{2}}{c^{2}}\right) \approx\left[1+\left(\frac{V_{P} 2 \sqrt{2} L}{\pi d_{o} c_{L}}\right)^{2}\right] \\
& \approx\left[1+0.81\left(\frac{L}{d_{o}}\right)^{2}\left(\frac{V_{P}}{c_{L}}\right)^{2}\right] .
\end{aligned}
$$

That is, a $100 \mathrm{~m}$ span would amplify the $1 \mathrm{~s}$ spectral acceleration of a $350 \mathrm{~m} / \mathrm{s}$ pod by a factor of about 4.24 . Notice that this amplification grows rapidly with the length of the span; a $200 \mathrm{~m}$ span would be a natural resonance at about $4 \mathrm{~s}$ and the corresponding 4-s spectral acceleration would be amplified by a factor of about 11.5. It appears that long unsupported spans of the pipe may have the greatest potential to produce large pod accelerations. As an example of a worst-case scenario, consider a 230-m-long bridge that consists of a pipe that is supported at its ends and that is subjected to the motions recorded at station KATNP during the 25 April 2015 M 7.8 Gorkha, Nepal, earthquake (Galetzka et al., 2015). A 230-m span length would produce a 5-s natural period with a corresponding amplification of spectral acceleration of about 17. The KATNP site recorded a 5-s spectral acceleration of about $0.5 \mathrm{~g}$, so the expected acceleration of a $350 \mathrm{~m} / \mathrm{s}$ pod that crosses the bridge when it was experiencing maximum deformation is about $8.5 \mathrm{~g}$, provided the system remains linear.

\section{Control Strategy to Minimize Inertial Forces on a Bridge}

Notice that the pod could be so lucky as to cross the bridge just as the tube is approximately straight. A $350 \mathrm{~m} / \mathrm{s}$ pod would require only $0.66 \mathrm{~s}$ to cross a 230 -m-long bridge that vibrates with an approximate period of $5 \mathrm{~s}$. The bridge is approximately straight when $\omega t=(n+(1 / 2)) \pi$ in equation (18), and then there is no lateral acceleration from the fundamental mode. This suggests a scheme that adjusts the speed of the pod such that the pod reaches the center of the bridge when the tube is temporarily straight. If the pod speed is adjusted so that it enters the bridge at the appropriate time, there should be no lateral acceleration due to resonance of the bridge. If there is an accelerometer at the center of the bridge that records the acceleration in the transverse directions to the bridge, the record could be integrated with respect to time to obtain the bridge particle velocity with time. If the maximum bridge velocities occur at times $t_{n}=t_{0}+(n / 2) T_{0}$, in which $T_{0}$ is the bridge fundamental period, and if the pod is initially traveling at velocity $V_{P}$, and if a braking deceleration $A$ is applied at time $t_{B}$ and distance $x_{B}$ from the center of the bridge, the position of the pod will be

$$
x(t)=x_{B}-V_{P}\left(t-t_{B}\right)-\frac{1}{2} A\left(t-t_{B}\right)^{2} .
$$

I can determine the deceleration $A$ such that the pod is at the bridge center when it is straight by solving

$$
\begin{aligned}
x\left(t=t_{n}\right)= & 0=x_{B}-V_{P}\left(t_{0}-t_{B}+\frac{n}{2} T_{0}\right) \\
& -\frac{1}{2} A\left(t_{0}-t_{B}+\frac{n}{2} T_{0}\right)^{2}
\end{aligned}
$$

or

$$
A=2 \frac{x_{B}-V_{P}\left(t_{0}-t_{B}+\frac{n}{2} T_{0}\right)}{\left(t_{0}-t_{B}+\frac{n}{2} T_{0}\right)^{2}} .
$$

Obviously, it is advantageous to send a message to the pod earlier, rather than later. The required accelerations decrease with the distance that the pod is from the bridge.

\section{Discussion}

I will admit that I was skeptical when Brogan BamBrogan came to Caltech to argue for the viability of a hyperloop system in California. The thought of high-speed motion through a pipe deforming in earthquakes seemed alarming. However, the simple analysis of this Short Note seems to indicate that the dynamic challenges are manageable. That said, I do not recommend using the analysis in this note for engineering design decisions; finite-element simulations of more realistic descriptions of the Earth are undoubtedly better tools for these problems. Nevertheless, this simple analysis can provide an insight into this problem.

\section{Data and Resources}

No data were used in this Short Note.

\section{Acknowledgments}

The author is grateful to an anonymous reviewer for helpful comments and for careful review of the equations, including several important corrections.

\section{References}

Galetzka, J., J.-P. Avouac, J. Genrich, and K. Hudnut (2015). Slip pulse and resonance of Kathmandu basin during the $2015 M_{\mathrm{w}} 7.8$ Gorkha earthquake, Nepal imaged with geodesy, Science doi: 10.1126/science. aac6383.

Garber, M. (2012). The real iPod: Elon Musk's wild idea for a "Jetson Tunnel" from S.F. to L.A., The Atlantic, July 13.

Honegger, D., D. Nyman, E. Johnson, L. Cluff, and S. Sorensen (2004). Trans-Alaska pipeline system performance in the 2002 Denali fault, Alaska, earthquake, Earthq. Spectra 20, 707-738.

Housner, G. W, and T. Vreeland Jr. (1965). The Analysis of Stress and Deformation, California Institute of Technology, Pasadena, California, 440 p.

Department of Mechanical and Civil Engineering

California Institute of Technology

MC 104-44

Pasadena, California 91125

heaton@caltech.edu

Manuscript received 17 February 2017; Published Online 25 September 2017 